\title{
New Paradigms in Cardiac Amyloidosis: The Current Experience of the Northeast Region of Brazil
}

\author{
Tonnison Silva, ${ }^{1,2}$ Carlos Eduardo Lucena Montenegro, ${ }^{3,4}$ Marcelo Dantas Tavares de Melo, ${ }^{\circledR}$ Luiz Ritt, ${ }^{1,2} \bullet$
} André Luiz Cerqueira Almeida ${ }^{\circledR}$

Escola Bahiana de Medicina e Saúde Pública, ${ }^{1}$ Salvador, BA - Brazil

Instituto D'or de Pesquisa e Ensino, Hospital Cárdio Pulmonar, ${ }^{2}$ Salvador, BA - Brazil

PROCAPE (Pronto Socorro Cardiológico de Pernambuco)/UPE (Universidade de Pernambuco), ${ }^{3}$ Recife, PE - Brazil

Real Hospital de Beneficência Portuguesa de Pernambuco, ${ }^{4}$ Recife, PE - Brazil

Universidade Federal da Paraíba (UFPB), ${ }^{5}$ Cidade Universitária, PB - Brazil

Santa Casa de Misericórdia de Feira de Santana, ${ }^{6}$ Feira de Santana, BA - Brazil

Cardiac amyloidosis (CA) is a severe and progressive infiltrative disease caused by the deposition of amyloid fibrils, with poor prognosis and limited therapeutic options. It can occur due to rare genetic variants or as a consequence of acquired conditions. Thanks to advances in imaging techniques and the possibility of obtaining non-invasive diagnosis, we now know that CA would perhaps be better classified as an underdiagnosed disease rather than as a rare one. ${ }^{1-3}$

González-Lopez et al. demonstrated that, in a series of 120 patients with preserved ejection fraction heart failure, $13.3 \%$ had a diagnosis of transthyretin-associated amyloid cardiomyopathy (ATTR-CM). ${ }^{4}$ In patients with aortic stenosis, we found a prevalence of around $15 \%$ of ATTR-CM, ${ }^{5}$ and, in necropsy studies, amyloid protein deposition was found in $25 \%$ of patients over 85 years old, which shows the importance of this diagnosis in the spectrum of myocardial diseases. ${ }^{6}$

Bone scintigraphy with PYP- ${ }^{99 \mathrm{~m}} \mathrm{TC}$ is being increasingly used in the diagnosis of ATTR-CM. ${ }^{7}$ Thus, it is expected that the number of patients diagnosed with ATTR-CM will continue to increase. New therapies reduce the progressive morbidity and mortality associated with ATTR-CM, contributing to improve the journey of these patients. The importance of early recognition of ATTR-CM is essential, as the prompt initiation of appropriate treatment is essential to maximize its therapeutic potential and improve patients' quality of life. ${ }^{8}$

As it presents heterogeneous clinical manifestations that may involve different medical specialties, the creation of groups dedicated to the identification and monitoring of these patients is of great value. ${ }^{2,9}$ In Brazil, Centers of Excellence (CEs) in amyloidosis have already been created, offering extensive care based on the team's experience with ATTR-CM, multidisciplinary approach, advanced diagnostics, and time dedicated to patient care and education.

\section{Keywords}

Amyloidosis; Prealbumin; Brazil.

Mailing Address: Carlos Eduardo Lucena Montenegro •

Universidade de Pernambuco - Miocardiopatias/ Transplante cardíaco

Rua dos Palmares, S/N. Postal Code: 50100-010, Recife, PE - Brazil

E-mail: ce montenegro@yahoo.com.br

Manuscript received September 06, 2021, revised manuscript

September 24, 2021, accepted September 24, 2021

DOI: https://doi.org/10.36660/abchf.20210013
Raising awareness of best practices in amyloidosis centers among health professionals can reinforce the benefits of early referral and comprehensive care for patients with ATTR-CM. ${ }^{10,11}$ However, as Brazil is a continent-sized country with many disparities related to public and private health systems, the different geographic regions have progressed separately in the creation of these centers. Recently, we brought together specialists from the Northeast Region of Brazil to share best practices and define the best flow for identification and management of ATTR-CM in institutions from different states. Here, we share our learning, difficulties faced (Table 1), and general point of view regarding the current scenario in our region.

One of the main difficulties in the setting of the Brazilian Unified Health System (SUS) is the structure to adequately complete the flowchart for non-invasive diagnosis of CA. The first obstacle we face, regardless of region, is the need to exclude immunoglobulin light-chain amyloidosis. Specific laboratory tests (immunofixation of proteins and kappa and lambda chain assays) are not covered by the SUS or even by some private health plans. Also regarding the setting of the SUS, pyrophosphate scintigraphy, echocardiography with myocardial strain analysis, and cardiac resonance are not performed in most tertiary cardiac care units in the Northeast Region. When we are with a patient who has been diagnosed with ATTR, it is necessary to distinguish whether it is the variant or senile form, and we then need to turn to genotyping, which can often only be done through partnerships with laboratories.

Before carrying out the genetic study and, subsequently, due to the size of the result, adequate genetic counseling is required,

Table 1 - Main difficulties faced, in different centers in the Northeast, in the search for specialized care for ATTR-CM

1.1 - Structural limitations to achieving diagnosis in a non-invasive manner

1.2 - Lack of basic medical education dedicated to the subject;

1.3 - Difficulties in connecting specialties to discuss the same case;

1.4 - Restricted access to genetic tests and interaction with geneticists;

1.5 - Difficulty in using the specific drug (tafamidis) that has been approved in Brazil, as well as others that have not yet been approved by the Brazilian Ministry of Health;

1.6 - Overload of public services, making it difficult to monitor diagnosed patients' family members. 
and it is at this point that the absence of a geneticist becomes evident. In supplementary medicine, we find fewer difficulties in this flow, which leads to a much faster diagnostic process, but the paths are no less tortuous, as the scarcity of geneticists and the high cost of genetic testing, in addition to the fact that they are not covered by supplementary health plans also hinder this diagnosis. The lack of interaction between specialists from different areas and the lack of basic and continuing education about ATTR-CM are also other important obstacles. It is noteworthy that, in the Northeast Region, there are already reference cardiology centers that have become CEs, with clinical support from an ophthalmologist, nephrologist, neurologist, and geneticist, but this number is still very small given the importance and increasing incidence of CA. The few people who have been involved in this area are, in a selfless and dedicated way, growing together and trying to develop diagnosis, in addition to spreading knowledge, but there is still a lot to be done. For example, there is still no widespread diffusion of the red flags that suggest diagnosis (Table 2), which would greatly facilitate the journey of these patients to their final diagnosis.

Despite all the barriers found, our region has stood out due to the growing number of diagnoses of this disease, making it possible for more patients to benefit from specific therapy. CEs in the region have been gaining expertise in the treatment of these patients, highlighting the symptomatic benefit of diuretic therapy and the poor tolerance of beta-blockers and reninangiotensin-aldosterone system blockers, confirming what has already been seen in other centers around the world. The use of direct anticoagulants in FA + cardiac amyloidosis has also been part of our practice, with good results, in terms of both efficacy and safety

The use of tafamidis, a drug that stabilizes the transthyretin protein, has been shown to reduce in mortality in patients with ATTR-CM, but its extremely high cost and its availability through the SUS is perhaps currently the biggest obstacle for the treatment of these patients. Once again, this is not a problem exclusive to the Northeast Region, but rather to the whole of Brazil and even in the most developed countries. Its use, via the SUS, is only authorized in association with family amyloidotic polyneuropathy (FAP), which, to a certain extent, restricts its administration. Furthermore, when doses of tafamidis versus placebo were evaluated in the ATTR-ACT ${ }^{12}$ and ATTR-ACT extension ${ }^{13}$ studies, it was possible to verify that the highest dose (80 mg) brought better results in worse outcomes. This posology, which is already on the medication package insert, is 4 times

Table 2 - "Red flags"

\begin{tabular}{l}
\hline When we should think about cardiac amyloidosis: \\
\hline 1 - Patients over 60 years old with heart failure with preserved \\
ejection fraction \\
\hline 2 - LVH of unclear etiology \\
\hline 3 - Intolerance or refractoriness to conventional treatment for heart failure \\
\hline 4 - History of carpal tunnel syndrome, mainly if bilateral \\
\hline 5 - Patients with polyneuropathy or family history of polyneuropathy \\
\hline 6 - Orthostatic hypotension and renal dysfunction with proteinuria \\
\hline
\end{tabular}

higher than that approved for the treatment of FAP and released by the SUS, which can bring major limitations in relation to therapy in ATTR-CM. The cost of tafamidis is undoubtedly a major limiting factor to its use; however, when we analyze the cost-effectiveness ratio, keeping in mind that the denominator is the main determinant in this ratio, we realize that the use of this drug is much more than indicated..$^{12,13}$ Despite all these obstacles, we have been able to use the drug, and it appears to be safe, with good results so far. We also remember that new drugs, such as patisiran and inotersen, must reach the market and can be approved by the SUS and that solving the problems of access to tafamidis will also help with greater accessibility to these drugs, when available.

Amyloidosis is potentially severe and progressive, and its incidence increases as we seek to further diagnose it. We, from the CEs in the Northeast Region, seek to offer the best and most up-to-date care to our patients with CA, and, despite the limitations, obstacles, and difficulties, we can say that in recent years, we have seen much more progress than setbacks, and this is precisely what encourages us to continue. The union of CEs, which are already centers of excellence in cardiology, is only the first step towards joint technical and scientific development, in order to overcome the difficulties intrinsic to our health system and also the disease itself, reaching the final goal, namely, the best care for our patients.

We have no doubt that many of the difficulties faced by CEs in our region are also shared by colleagues throughout Brazil, and it is through further discussion and approaches to the topic, as we are doing through this publication, that we will ensure that more people are diagnosed early, that genetic counseling (when indicated) is done, and that patients are treated in accordance with international recommendations. ${ }^{3}$

\section{Author contributions}

Writing of the manuscript: Silva T, Montenegro CEL, Melo MDT, Ritt L, Almeida ALC.Critical revision of the manuscript for intellectual content: Silva T, Montenegro CEL, Melo MDT, Ritt L, Almeida ALC.

\section{Potential Conflict of Interest}

No potential conflict of interest relevant to this article was reported.

\section{Sources of Funding}

There were no external funding sources for this study.

\section{Study Association}

This study is not associated with any thesis or dissertation work.

\section{Ethics approval and consent to participate}

This article does not contain any studies with human participants or animals performed by any of the authors. 


\section{References}

1. Maurer MS, Elliott P, Comenzo R, Semigran M, Rapezzi C. Addressing Common Questions Encountered in the Diagnosis and Management of Cardiac Amyloidosis. Circulation. 2017;135(14):1357-77. doi: 10.1161/ CIRCULATIONAHA.116.024438.

2. Ruberg FL, Grogan M, Hanna M, Kelly JW, Maurer MS. Transthyretin Amyloid Cardiomyopathy: JACC State-of-the-Art Review. J Am Coll Cardiol. 2019;73(22):2872-91. doi: 10.1016/j.jacc.2019.04.003.

3. Garcia-Pavia P, Rapezzi C, Adler Y, Arad M, Basso C, Brucato A, et al. Diagnosis and Treatment of Cardiac Amyloidosis: A Position Statement of the ESC Working Group on Myocardial and Pericardial Diseases. Eur Heart J. 2021;42(16):1554-68. doi: 10.1093/eurheartj/ehab072.

4. González-López E, Gallego-Delgado M, Guzzo-Merello G, Haro-Del Moral FJ, Cobo-Marcos M, Robles C, et al. Wild-Type Transthyretin Amyloidosis as a Cause of Heart Failure with Preserved Ejection Fraction. Eur Heart J. 2015;36(38):2585-94. doi: 10.1093/eurheartj/ehv338.

5. Scully PR, Treibel TA, Fontana M, Lloyd G, Mullen M, Pugliese F, et al. Prevalence of Cardiac Amyloidosis in Patients Referred for Transcatheter Aortic Valve Replacement. J Am Coll Cardiol. 2018;71(4):463-4. doi: 10.1016/j.jacc.2017.11.037

6. Tanskanen M, Peuralinna T, Polvikoski T, Notkola IL, Sulkava R, Hardy J, et al. Senile Systemic Amyloidosis Affects 25\% of the Very Aged and Associates with Genetic Variation in Alpha2-Macroglobulin and Tau: A Population-Based Autopsy Study. Ann Med. 2008;40(3):232-9. doi: 10.1080/07853890701842988.
7. Rezk T, Fontana M, Gillmore JD. A Review of the Criteria for Non-Invasive Diagnosis of Cardiac Transthyretin Amyloidosis. Expert Opin Orphan Drug. 2021;9(3):87-94. doi: 10.1080/21678707.2021.1898371.

8. Lindmark K, Pilebro B, Sundström T, Lindqvist P. Prevalence of Wild Type Transtyrethin Cardiac Amyloidosis in a Heart Failure Clinic. ESC Heart Fail. 2021;8(1):745-9. doi: 10.1002/ehf2.13110.

9. Witteles RM, Bokhari S, Damy T, Elliott PM, Falk RH, Fine NM, et al Screening for Transthyretin Amyloid Cardiomyopathy in Everyday Practice. JACC Heart Fail. 2019;7(8):709-16. doi: 10.1016/j.jchf.2019.04.010

10. Nativi-Nicolau J, Sarswat N, Fajardo J, Finkel M, Abdulsattar Y, Castaño A, et al. Best Practices in Specialized Amyloidosis Centers in the United States: A Survey of Cardiologists, Nurses, Patients, and Patient Advocates. J Clin Res Pediatr Endocrinol. 2021:13(2):124-35. doi: 10.1177/11795468211015230.

11. Dang D, Fournier P, Cariou E, Huart A, Ribes D, Cintas P, et al. Gateway and Journey of Patients with Cardiac amyloidosis. ESC Heart Fail. 2020;7(5):2418-30. doi: 10.1002/ehf2.12793.

12. Maurer MS, SchwartzJH, Gundapaneni B, Elliott PM, Merlini G, WaddingtonCruz M, et al. Tafamidis Treatment for Patients with Transthyretin Amyloid Cardiomyopathy. N Engl J Med. 2018;379(11):1007-16. doi: 10.1056/ NEJMoa1805689.

13. Damy T, Garcia-Pavia P, Hanna M, Judge DP, Merlini G, Gundapaneni B, et al. Efficacy and Safety of Tafamidis Doses in the Tafamidis in Transthyretin Cardiomyopathy Clinical Trial (ATTR-ACT) and Long-Term Extension Study. Eur J Heart Fail. 2021;23(2):277-85. doi: 10.1002/ejhf.2027. 